\title{
TUBE BILLET'S SHAPING ON THE PRE-SHAPING PRESS BY MEANS OF PUNCH WITH FLAT BOTTOM
}

\author{
V. N. Shinkin ${ }^{1}$ \\ ${ }^{1}$ National University of Science and Technology “MISiS” (Moscow, Russia)
}

E-mail: shinkin-korolev@yandex.ru

$\begin{array}{ll}\text { A U T H O R ' S I N F O A B S T R A C T } \\ \begin{array}{ll}\text { V. N. Shinkin, } \\ \begin{array}{l}\text { Dr. Phys.-Math., } \\ \text { Professor }\end{array}\end{array} \\ \begin{array}{l}\text { The shaping of the large-diameter pipes on the pre-shaping press of the tube-electric welding unit with help of the punch } \\ \text { with a flat-bottom is an important applied technical problem in the ferrous metallurgy. The proper selection of the shape's } \\ \text { characteristics of the punch and the shaping's characteristics for the given characteristics of the steel sheet is the complicated } \\ \text { technical problem for the technologists of the pipe shops. On the one hand, the insufficient reduction of the steel billet on } \\ \text { the pre-shaping press leads to too large a gap between the longitudinal edges of the steel sheet. In this case, the welding of the } \\ \text { sheet edges is impossible. The pipe billet has to be additional bent on the special press before the welding of the sheet's edges, } \\ \text { since there is not enough power of the devices for pressing the edges to each other on the electric welding unit before the pipe's } \\ \text { assembling. On the other hand, the excessive reduction of the steel billet on the pre-shaping press leads to the defect of the } \\ \text { punch with flat bottom, } \\ \text { tube-electric welding } \\ \text { aggregation. }\end{array} & \begin{array}{l}\text { edges' overcloak after shaping the billet on the final-shaping press. All this leads to significant downtimes in the continuous } \\ \text { manufacture process of the steel pipes. }\end{array}\end{array}$

\section{Introduction}

The article deals with the shaping of steel billets for the large-diameter pipes on the tube-electric welding aggregation 1020 (TEWA 1020). The main mechanical processes of pipe shaping can be divided into three technological stages. The first mechanical stage is shaping the longitudinal edges of the steel sheet blank on the edge bending press (the flanging press). The second mechanical stage is shaping of the central part of the steel billet on the pre-shaping press (the U-press). The third mechanical stage is the shaping of the steel billet on the final-shaping press (the O-press). During the shaping, the upper and lower semi-cylindrical working surfaces of the $\mathrm{O}$-press get closer to each other, giving the billet the $\mathrm{O}$-shape.

The main factor at shaping the pipe billet, which answers for the curvature's radius of the billet during its bending and after its back-springing, is the bending moment $M$ of the sheet's cross-section [1-4]. There are the various ways to analytically approximate the steel's hardening zone from the yield strength to the ultimate strength. Each method has its own analytical expression for the bending moment. The more accurate the approximation of the steel's hardening zone, the more accurately the bending moments and residual radii of curvature of the pipe's billet are calculated after its elastic-plastic shaping on the presses [5-10].

The main mechanical characteristics (properties) of steel billets are the young modulus $E$, the yield strength $\sigma_{y}$, the ultimate strength $\sigma_{u}$, the relative elongation $\delta$ at break, the relative narrowing $\psi$ at break, the modulus hardening $P_{y}$ in the yield strength, the relative elongation $\varepsilon_{y}=\sigma_{y} / E$ in the yield strength, the relative elongation $\varepsilon_{u}$ in the ultimate strength (at the moment of neck's formation) [11].

Below we will use the direct cubic approximation of the steel's hardening zone

$$
\begin{aligned}
& \sigma(\varepsilon)=\left(E \varepsilon, 0 \leq \varepsilon \leq \varepsilon_{y}=\frac{\sigma_{y}}{E} ; \sigma_{\text {plastic }}(\varepsilon), \varepsilon_{y}<\varepsilon \leq \varepsilon_{u}\right), \\
& \sigma_{\text {plastic }}(\varepsilon)=\sigma_{y}+P_{y}\left(\varepsilon-\varepsilon_{y}\right)-\frac{2 P_{y}\left(\varepsilon_{u}-\varepsilon_{y}\right)-3\left(\sigma_{u}-\sigma_{y}\right)}{\left(\varepsilon_{u}-\varepsilon_{y}\right)^{2}} \times \\
& \times\left(\varepsilon-\varepsilon_{y}\right)^{2}+\frac{P_{y}\left(\varepsilon_{u}-\varepsilon_{y}\right)-2\left(\sigma_{u}-\sigma_{y}\right)}{\left(\varepsilon_{u}-\varepsilon_{y}\right)^{3}}\left(\varepsilon-\varepsilon_{y}\right)^{3},
\end{aligned}
$$

where $\sigma$ is the normal stress, $\varepsilon$ is the relative elongation.

The steel's hardening curve passes through the yield strength and the ultimate strength, and also has the maximum in the ultimate strength:

$$
\sigma\left(\varepsilon_{y}\right)=\sigma_{y}, \sigma\left(\varepsilon_{u}\right)=\sigma_{u}, \frac{d \sigma\left(\varepsilon_{y}\right)}{d \varepsilon}=P_{y}, \frac{d \sigma\left(\varepsilon_{u}\right)}{d \varepsilon}=0 .
$$

The modulus hardening $P_{y}$ in the yield strength and the relative elongation $\varepsilon_{u}$ in the ultimate strength are easily determined using the stretching diagram of steel [12-17]. If there is no the steel's stretching diagram, then for the high-strength pipe steels, the values of $P_{y}$ and $\varepsilon_{u}$ can be determined fairly accurately by the approximate formulas:

$$
\varepsilon_{y}=\frac{\sigma_{y}}{E}, \quad \varepsilon_{u} \approx \delta(1-\psi), \quad P_{y} \approx \frac{2\left(\sigma_{u}-\sigma_{y}\right)}{\varepsilon_{u}-\varepsilon_{y}} .
$$

The values of the elongation $\delta$ at break and the relative narrowing $\psi$ at break can be found in any marochnik of steel [16-21].

The main geometrical characteristics at the steel sheet's shaping (the initial pipe's billet) are the thickness $h$, the width $b$, the curvature $k$ of the longitudinal median (neutral) line and the curvature's radius $\rho=1 / k[1,2,11]$. 
At $\rho \geq \rho_{y}=E h /\left(2 \sigma_{y}\right)$ is observed the purely elastic bending the flat steel sheet, and at $\rho<\rho_{y}=E h /\left(2 \sigma_{y}\right)$ is observed the elastoplastic bending the flat steel sheet [12].

The dimensionless bending moment in the sheet's cross-section, corresponding to the direct cubic approximation of the steel's hardening zone, has the form

$$
\begin{aligned}
& F(S h i)=\frac{12 M}{b h^{2} \sigma_{y}}, \quad S h i=\frac{E h}{\sigma_{y} \rho}, \quad S h i\left(\rho_{y}\right)=2, \\
& F(S h i)=\left(S h i, \quad 0 \leq S h i \leq 2 ; \quad F_{\text {plastic }}(S h i), \quad \text { Shi }>2\right), \\
& F_{\text {plastic }}(S h i)=3-\frac{4}{S h i^{2}}+\frac{P_{y}}{E} S h i\left(1-\frac{2}{S h i}\right)^{2}\left(1+\frac{1}{S h i}\right)- \\
& -\frac{2 P_{y}\left(\varepsilon_{u}-\varepsilon_{y}\right)-3\left(\sigma_{u}-\sigma_{y}\right) \sigma_{y}}{4 E^{2}\left(\varepsilon_{u}-\varepsilon_{y}\right)^{2}} \operatorname{Shi}^{2}\left(1-\frac{2}{S h i}\right)^{3}\left(\frac{3}{2}+\frac{1}{S h i}\right)+ \\
& +\frac{3 \sigma_{y}{ }^{2}\left(P_{y}\left(\varepsilon_{u}-\varepsilon_{y}\right)-2\left(\sigma_{u}-\sigma_{y}\right)\right)}{40 E^{3}\left(\varepsilon_{u}-\varepsilon_{y}\right)^{3}} S h i^{3}\left(1-\frac{2}{S h i}\right)^{4}\left(2+\frac{1}{S h i}\right),
\end{aligned}
$$

where Shi is the dimensionless criterion of the curvature of the longitudinal median line of sheet.

\section{Bending a steel sheet on the flanging press}

There are possible two variants for bending the edges of the thick steel sheet - the bending of the sheet's edges simultaneously on both sides (the first variant) and the bending of the sheet's edges on each side separately (the second variant) $[3,9,11,12]$.

On the flanging press by SMS Meer of the electricwelded tube complex for big-diameter tube, the edges of the sheet are bent simultaneously from both sides. However, the length of the bent edge is significantly less (in several times) than the total length of the steel sheet. Therefore, the operation of edges' bending repeats the several times along the sheet's length. The advantage of this variant of edges' bending is the ability to deform the very thick steel sheet (up to $48 \mathrm{~mm}$ ) with the high ultimate strength of the metal (up to $650 \mathrm{MPa}$ ). The disadvantage of this variant is the possibility of shaping the corrugations of the edges along the sheet's length. In this case, the sheet's edge has the small fold or unevenness, which is practically impossible to eliminate during the subsequent technological operations of the pipe's production from steel sheet. Therefore, the high-quality welding of the opposite edges of the sheet is impossible.

On the flanging press by TEWA 1020, the longitudinal edge of the steel sheet is bent according to the two-radius scheme along the entire length of the sheet, first on one side, and then on the opposite side (see fig. 1). The corrugation of the sheet's edge is not formed in this case. However, the maximum possible thickness of the sheet with a high ultimate strength of the metal in this case is much less (up to $32 \mathrm{~mm}$ ).

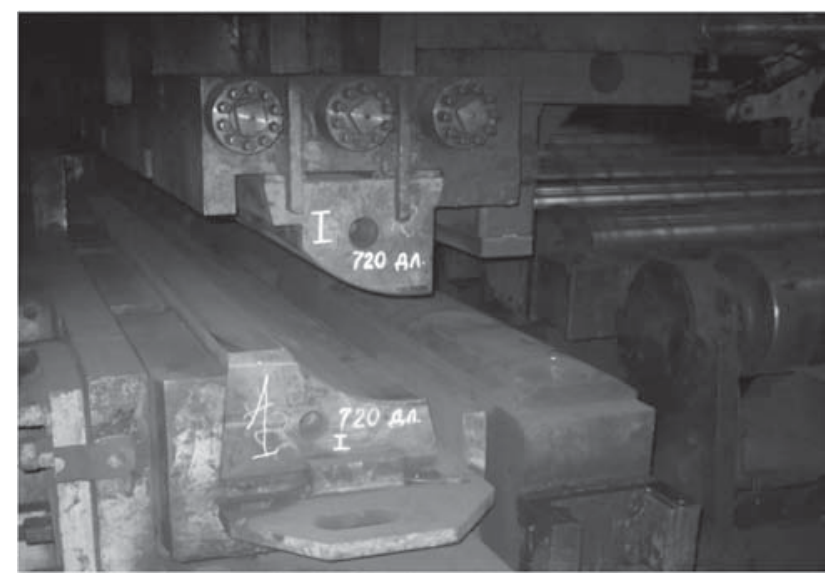

Fig. 1. The flanging press for steel sheet and its punch and matrix

Let $R_{e i}$ and $\varphi_{e i}(i=1,2)$ be the radius and angle of bending of the $i$-th zone of the die's outer surface of the flanging press (fig. 1), and $S_{e}$ be the length of the bent edge of the sheet on one side.

At bending the edges of the sheet, the side edge of the sheet is located inside the second zone $(i=2)$ of the matrix, and the curvature of the $i$-th zone of the neutral line of the sheet's bent edge is $\varepsilon_{e i}=1 /\left(R_{e i}+h / 2\right)$. The length of the first zone of the sheet edge is $S_{56}=S_{e 1}=\left(R_{e 1}+h / 2\right)$ $\varphi_{e 1}$, and the length of the second zone of the sheet edge is $S_{67}=S_{e 2}=S_{e}-S_{e 1}$.

\section{Bending a steel sheet on the pre-shaping press}

In the shaping process on the pre-shaping press (fig. 2), the steel billet is first bent by the punch of the vertical bending beam, and then by the side rollers of the horizontal bending beam.

The punch can be of two main types -- the semi-circular punch with the side sloping flat inserts and the semicircular punch with a flat bottom. Each type of punch has its own advantages and disadvantages. Shaping with the punch with side sloping flat inserts was considered earlier in one of the author's article. In this paper, we study the shaping of steel sheet with the semi-circular punch with a flat bottom. One of the advantages of shaping by the semi-

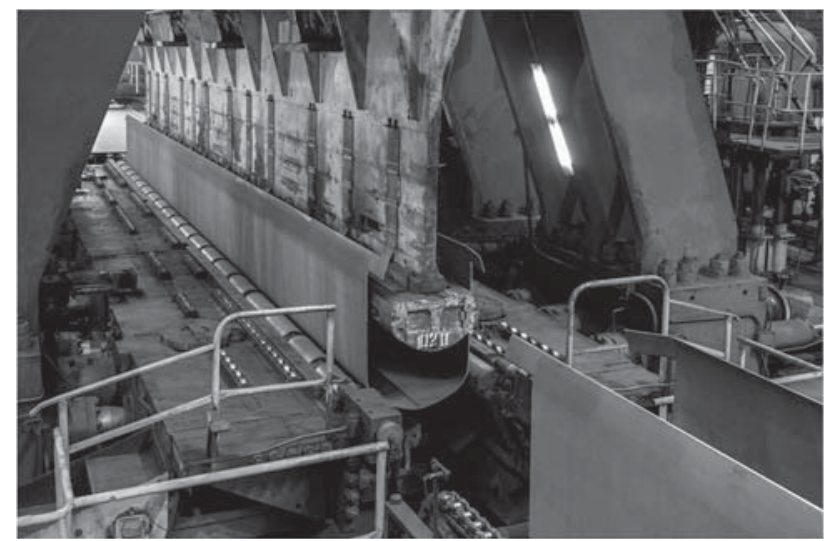

Fig. 2. The pre-shaping press TEWA 1020 for bending the central region of steel sheet 
circular punch with a flat bottom is the stability of the steel billet during the shaping on the U-press. The contact surface of the punch with the sheet is maximum in this case. Consequently, the friction forces, that prevent shifting the sheet during the shaping, are maximum. Therefore, the lateral displacement of the sheet during the shaping is minimal. Hence, the probability of displacement of the longitudinal edges of the pipe billet relative to the sheet's longitudinal axis of symmetry is minimal.

The displacement of the sheet's longitudinal edges relative to each other is the frequent defect, that is difficult to eliminate during the shaping the pipe. The defect of the edges' displacement leads to the poor-quality weld (the weld defect) during the assembling the pipe in the pipe welding unit.

On the pre-shaping press, the steel billet is shaped to the U-shape by bending its central part. The shape of the cross-section of the punch with a flat bottom is shown in fig. 3. The punch has a vertical axis of symmetry. The punch's section 0-1 (the half-width of the flat bottom) is a straight segment with the length $l$, the punch's sections 1-2 and 2-3 are the circular arcs with the radius $R_{p}$.

The point 3 and the angle $\alpha_{c}$ correspond to the point of separation of the steel billet from the punch. The billet's section $0-1$ (the half-width of the flat bottom) is a straight segment with the length $S_{01}=l$. The billet's sections $1-2$ and 2-3 are the circular arcs with the radius $R_{p}+h / 2$ and curvature $\varepsilon_{p}=1 /\left(R_{p}+h / 2\right)$. The lengths of the sections 1-2 and 2-3 are correspondently equal to

$$
S_{12}=\left(R_{p}+\frac{h}{2}\right) \frac{\pi}{2}, \quad S_{23}=\left(R_{p}+\frac{h}{2}\right) \alpha_{c} .
$$

The angle of separation of the steel billet from the punch is equal to

$$
\begin{aligned}
& \alpha_{c}=\arcsin \left(\frac{R_{p}+R_{c}+h}{\sqrt{\left(R_{p}+R_{c}-H_{c}\right)^{2}+\left(L_{c}+R_{c}\right)^{2}}}\right)- \\
& -\operatorname{arctg}\left(\frac{R_{p}+R_{c}-H_{c}}{L_{c}+R_{c}}\right),
\end{aligned}
$$

where $H_{c}$ and $L_{c}$ are the characteristics of the billet's compression by the vertical punch and horizontal bending rollers, $R_{c}$ is the radius of the horizontal bending rollers.

The billet's sections 3-4 and 4-5 are the straight segments, and the billet's sections 5-6 and 6-7 correspond to the bending zones of the longitudinal edges of the steel sheet on the flanging press. The length of the straight segment 3-4 is equal to

$$
S_{34}=\frac{L_{c}+R_{c}}{\cos \alpha_{c}}-\left(R_{p}+R_{c}+h\right) \operatorname{tg} \alpha_{c} .
$$

The length of the straight segment 4-5 is equal to

$$
S_{45}=\frac{b-2 S_{01}-2 S_{12}-2 S_{23}-2 S_{34}-2 S_{e}}{2} .
$$

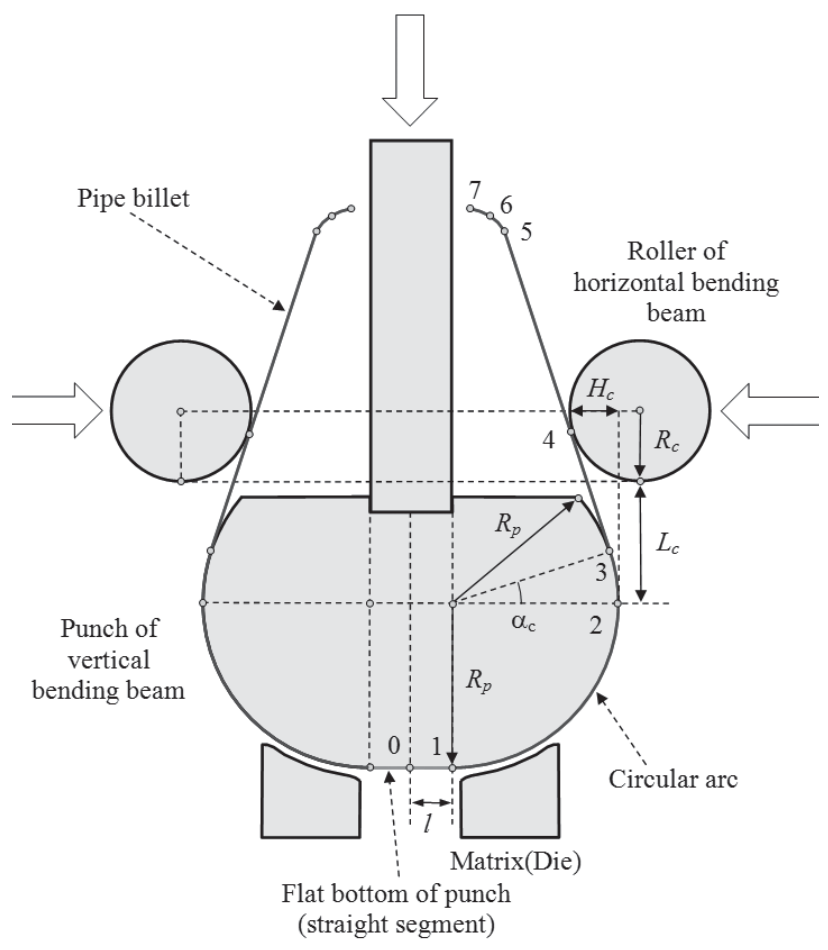

Fig. 3. Bending scheme of steel sheet on the pre-shaping press of TEWA 1020

The curvature of the straight segments $0-1,3-4$ and 4-5 is equal to zero.

We note that the areas of plastic deformation on the flanging press and on the U-press do not intersect.

If the wrong shape of the punch is chosen and the steel sheet is bent too much on the U-press, the pipe billet may hang on the press's punch, when the punch is lifted up, breaking away from the lower dies of the U-press.

\section{Bending a steel pipe billet on the final-shaping press}

On the final-shaping press, the cross-section of the steel pipe billet is given a circular shape due to its compression by the upper punch and the lower die with the cylindrical surfaces. The curvature's radius of the punch's inner surface of the O-press is $R_{f}$. The curvature of the neutral line of the pipe billet at the end of shaping on the O-press is equal to $\varepsilon_{f}=1 /\left(R_{f}-h / 2\right)$ (fig. 4).

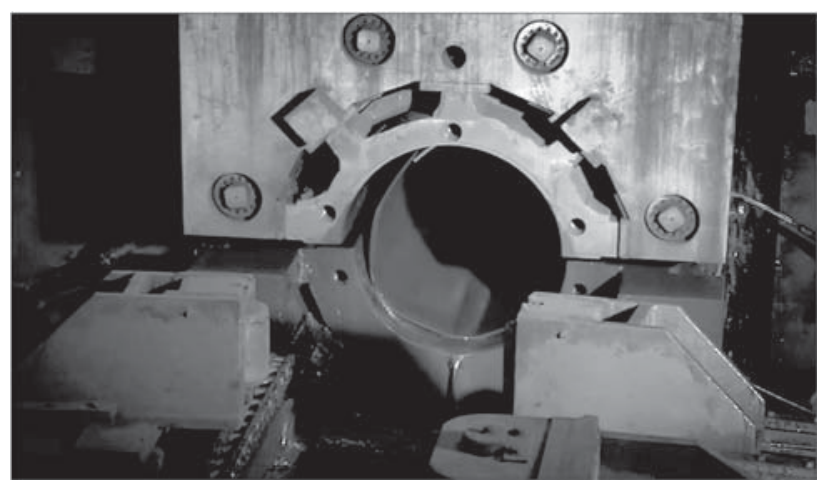

Fig. 4 Bending of steel pipe billet on the final-shaping press of TEWA 1020 
At shaping on the O-press, the excessively curved sections 1-2, 2-3, 5-6 and 6-7 of the steel billet are partially unbent in the opposite direction. The straight sections $0-1$, 3-4 and 4-5 are bending by the convexity in the direction to the contact surfaces of the punch and matrix of the O-press.

The sign-alternating elastic-plastic bending of a steel sheet and beam is an extremely difficult problem, both for the mechanical engineers and for the materials scientist technologists. In this case, we have to take into account the Hencky's theorem about the linear springing- back of the metal, the Masing's principle about the size of the elastic zone under the sign-alternating deformation, the Bauschinger's effect about the change of the metal's yield strength under the sign-alternating loading, and so on $[1-4,12,21]$. The scheme for calculating the curvature's radii of steel sheet with the sign-alternating bending of sheet's billet was repeatedly considered earlier by the author in a number of his works. Therefore, next we will not consider in detail the physical, mechanical and material science foundations of the signalternating bending of steel sheet, but will use the previously obtained the theoretical and experimental results.

\section{Calculation of residual radii of sheet's billet after sign-alternating bending in $\mathrm{O}$-press}

Note that for most the high-strength pipe steels, the hardening curves of bending moments for the positive and negative bending are anti-symmetrical if their initial points, corresponding to the ends of the elastic deformation zones under the stretching and compression will be combined with each other (the ideal Bauschinger's effect at bending).

According to the Hencky's theorem, the curvature's residual radii of the neutral line of the sections $0-1,3-4$ and 4-5 after shaping on the $\mathrm{O}$-press are calculated by the formulas:

$$
\begin{aligned}
& S h i_{f}=\frac{E h \varepsilon_{f}}{\sigma_{y}}=\frac{E h}{\sigma_{y}\left(R_{f}-\frac{h}{2}\right)}, \\
& S h i_{01 \text { end }}=S h i_{34 \text { end }}=S h i_{45 \text { end }}=S h i_{\text {fend }}=S h i_{f}-F\left(S h i_{f}\right),
\end{aligned}
$$

$$
\begin{aligned}
& \varepsilon_{01 \text { end }}=\varepsilon_{34 \text { end }}=\varepsilon_{45 \text { end }}=\varepsilon_{\text {fend }}=\frac{S h i_{\text {fend }} \sigma_{y}}{E h} \\
& R_{01 \text { end }}=R_{34 \text { end }}=R_{45 \text { end }}=R_{\text {fend }}=\frac{E h}{S h i_{\text {fend }} \sigma_{y}} .
\end{aligned}
$$

According to the Hencky's theorem, the Masing's principle, and the ideal Bauschinger's effect at bending, the curvature's residual radii of the neutral line of the sections 1-2 and 2-3 after shaping on the O-press are calculated by the formulas:

$$
S h i_{p}=\frac{E h \varepsilon_{p}}{\sigma_{y}}=\frac{E h}{\sigma_{y}\left(R_{p}+\frac{h}{2}\right)},
$$

$$
\begin{aligned}
& S h i_{12 \text { end }}=S h i_{23 \text { end }}=S h i_{\text {pend }}= \\
& =S h i_{f}+\left(F\left(S h i_{p}-2-S h i_{f}\right)+2-F\left(S h i_{p}\right)\right), \\
& \varepsilon_{12 \text { end }}=\varepsilon_{23 \text { end }}=\varepsilon_{\text {pend }}=\frac{S h i_{\text {pend }} \sigma_{y}}{E h}, \\
& R_{12 \text { end }}=R_{23 \text { end }}=R_{\text {pend }}=\frac{E h}{S h i_{\text {pend }} \sigma_{y}} .
\end{aligned}
$$

According to Hencky's theorem, the Masing's principle, and the ideal Bauschinger's effect at bending, the curvature's residual radii of the neutral line of the sections 5-6 and 6-7 after shaping on the O-press are calculated by the formulas:

$$
\begin{aligned}
& S h i_{e 1}=\frac{E h}{\sigma_{y}\left(R_{e 1}+\frac{h}{2}\right)}, \quad S h i_{e 2}=\frac{E h}{\sigma_{y}\left(R_{e 2}+\frac{h}{2}\right)}, \\
& S h i_{e \text { lend }}=S h i_{f}+\left(F\left(S h i_{e 1}-2-S h i_{f}\right)+2-F\left(S h i_{e 1}\right)\right), \quad(5) \\
& S h i_{e 2 e n d}=S h i_{f}+\left(F\left(S h i_{e 2}-2-S h i_{f}\right)+2-F\left(S h i_{e 2}\right)\right), \quad(6 \\
& R_{56}=R_{\text {elend }}=\frac{E h}{S h i_{\text {elend }} \sigma_{y}}, \quad R_{67}=R_{e 2 \text { end }}=\frac{E h}{S h i_{\text {e2end }} \sigma_{y}} .
\end{aligned}
$$

We note that at bending, the length of the neutral line of the sheet's cross-section does not change. Therefore, we can define the bending angle of the circular sections of the pipe billet as the ratio of their length to their curvature's radius. Knowing the radii and bending angles of the billet's sections, we can find the shape of this billet using the classical multiradius scheme of calculation.

Let the Young's modulus of steel $E=2 \times 10^{11} \mathrm{~Pa}$, the yield stress of steel $\sigma_{y}=525 \mathrm{MPa}$, the ultimate strength of steel $\sigma_{u}=612 \mathrm{MPa}$, the hardening modulus of steel at the yield stress $P_{y}=985.42 \mathrm{MPa}$, the relative elongation at the ultimate strength $\varepsilon_{u}=0.1792$, the relative elongation at break $\delta=0.32$, the relative narrowing at break $\psi=0.44$. Let the thickness and width of the steel sheet be correspondently $h=24 \mathrm{~mm}$ and $b=3.1353 \mathrm{~m}$. Let the curvature's radii and bending angles of the punch of the flanging press be correspondently $R_{e 1}=0.376 \mathrm{~m}, R_{e 2}=0.303 \mathrm{~m}, \varphi_{e 1}=23.5^{\circ}$, $\varphi_{e 2}=16.5^{\circ}$ and $S_{e}=0.2499 \mathrm{~m}$. Let the half-length of the width of the punch's flat bottom be $l=60 \mathrm{~mm}$. Let the radius of the U-press's punch be $R_{p}=0.32 \mathrm{~m}$, and the rollers' radius of the horizontal bending beam be $R_{c}=0.13 \mathrm{~m}$. Let the characteristic of the mutual vertical positions of the punch and bending rollers $L_{c}=0.284 \mathrm{~m}$, and the maximum compression of the steel billet $H_{c}=0.025 \mathrm{~m}$. Let the internal radius of the punch and matrix of the O-press $R_{f}=0.511 \mathrm{~m}$.

\section{Calculation of steel billet's shape for pipe with diameter of $1020 \mathrm{~mm}$}

Then $S_{01}=0.06 \mathrm{~m}, S_{12}=0.5215 \mathrm{~m}, S_{23}=0.0422 \mathrm{~m}$, $S_{34}=0.3569 \mathrm{~m}, S_{45}=0.3373 \mathrm{~m}, S_{56}=0.1591 \mathrm{~m}$, $S_{67}=0.0907 \mathrm{~m}, \alpha_{c}=7.2743^{\circ}, R_{01 \text { end }}=R_{34 \text { end }}=R_{45 \text { end }}=$ 
$=0.5991 \mathrm{~m}, R_{12 \text { end }}=R_{23 e n d}=0.4535 \mathrm{~m}, R_{56 e n d}=0.4604 \mathrm{~m}$, $R_{67 \text { end }}=0.4530 \mathrm{~m}$.

Knowing the curvature's final radii and the length of the steel billet's sections, we find the final shape of the billet after the O-press by means of the multi-radius scheme of calculation (fig. 5, curve 4). The width of the horizontal gap between the billet's edges after the O-press $\Delta_{O}=0.476 \mathrm{~mm}$, and the edges' height $H_{O}=1056.4 \mathrm{~mm}$. If the calculations do not take into account the Bauschinger's effect and the Masing's principle, then $\Delta_{O}=600.2 \mathrm{~mm}$ and $H_{O}=1117.5 \mathrm{~mm}$ (Fig. 5, dotted curve 5), which completely unsatisfactorily describes the actual final shape of the pipe's billet.

\section{Conclusions}

The new analytical method (1-6) for calculating the shape of a thick-walled steel sheet billet on three mechanical presses of the pipe-electric welding aggregation 1020 , including the pre-shaping press using the punch with a flat bottom, is proposed. The analytical simulation describes consecutive shaping the sheet metal during its shaping on the flanging press, the pre-shaping press and the final-shaping press. For describing the elastic and plastic properties of steel is used new analytical high-precision nonlinear dependence $(1,2)$ of the normal stress on the relative deformations, taking into account the yield strength, the ultimate strength and the hardening modulus at the yield strength. The residual radii of curvature at shaping the pipe billet are calculated with help of the new analytical dependence of the dimensionless bending moment on the sheet's curvature, the Hencky's theorem about the linear springing-back, the Masing's principle about the size of the elastic zone at the sign-alternating bending and the Bauschinger's effect about the change of the yield strength at the sign-alternating bending (3-6). It is shown that the numerical modeling, based on the constructed mathematical model and the multi-radius scheme of calculation, allows us to calculate with high accuracy the shape of the cross-section of the pipe billet after shaping on all three mechanical presses of the pipeelectric welding aggregation 1020.

\section{REFERENCES}

1. Shinkin V. N. Analytical description of metal plasticity at shift. CIS Iron and Steel Review. 2020. Vol. 19. pp. 66-70.

2. Shinkin V. N. Non-linear description of hardening zone of steel. Journal of Physics: Conference Series. 2020. Vol. 1431. 012069.

3. Grote K.-H., Hefazi H. Springer handbook of mechanical engineering. Springer. 2021. 1450 p.

4. Molotnikov V., Molotnikova A. Theory of elasticity and plasticity: A textbook of solid body mechanics. Springer. 2021. 485 p.

5. Belskiy S. M., Pimenov V. A., Shkarin A. N. Mathematical model for evaluating the actual form of the profile's contour of the hot-rolled strip's cross section. Journal of Chemical Technology and Metallurgy. 2021. Vol. 56. No. 1. pp. 214-220.

6. Belskiy S. M., Shopin I. I. On issue of strip's break during cold rolling. IOP Conference Series: Materials Science and Engineering. 2020. Vol. 969. 012072.

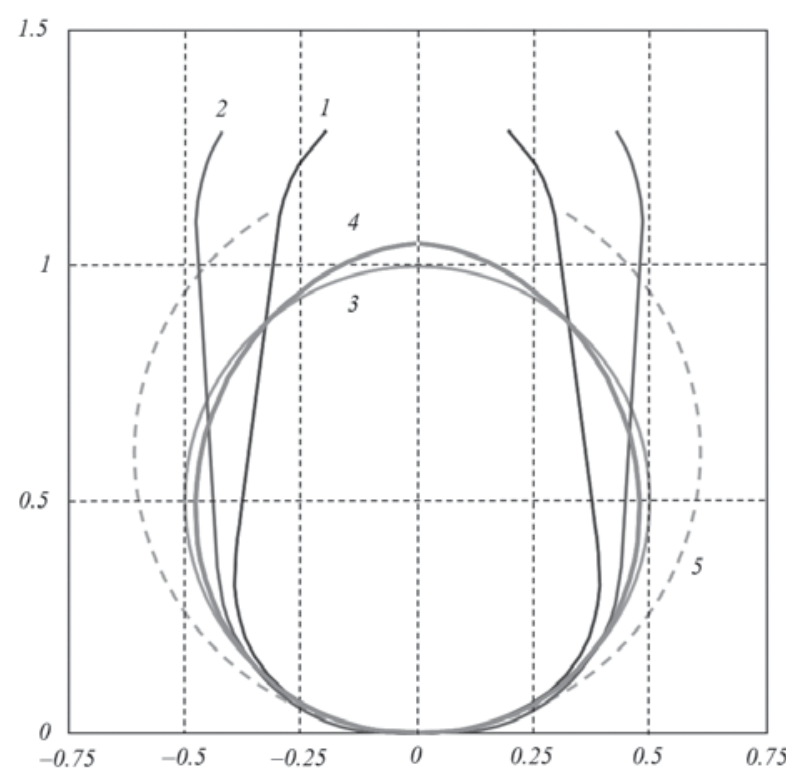

Fig. 5. Shape of steel pipe's billet at shaping on TEWA 1020: 1 - in the U-press; 2 - after the U-press; 3 - in the $\mathrm{O}$-press; 4 - after the $\mathrm{O}$-press (taking into account the Bauschinger's effect); 5 - after the O-press (not taking into account the Bauschinger's effect)

7. Tarantino A. M., Lanzoni L., Falope F. O. The bending theory of fully nonlinear beams. Springer. 2019. 87 p.

8. Ushakov I. V. Method of mechanical testing of laser treated metallic glass by indenters with different geometry. Proceedings of SPIE - The International Society for Optical Engineering. 2007. Vol. 6597. 659714.

9. Gasik M. Handbook of ferroalloys: Theory and technology. Butterworth-Heinemann. 2013. 536 p.

10. Jazar R. N. Approximation methods in science and engineering. Springer. 2020. 537 p.

11. Shinkin V. N. Torque at elastic-plastic torsion of sheet-straightening machines' steel shafts. Chernye Metally. 2020. No. 11. pp. 35-39.

12. Shinkin V. N. To question about moment at elasticoplastic bending. IOP Conference Series: Materials Science and Engineering. 2020. Vol. 971. 042055.

13. Cazacu O., Revil-Baudard B. Plasticity of metallic materials: Modeling and applications to forming. Elsevier. 2020. 560 p.

14. Li H., Fu M. Deformation-based processing of materials: Behavior, performance, modeling and control. Elsevier. 2019. 350 p.

15. Belskiy S. M., Shopin I. I. Statistical regression analysis of breakages in cold rolling. IOP Conference Series: Materials Science and Engineering. 2020. Vol. 969. 012074.

16. Ritchie R., Liu D. Introduction to fracture mechanics. Elsevier. 2021. $152 \mathrm{p}$.

17. Gonzalez-Velazquez J. L. Mechanical behavior and fracture of engineering materials. Springer. 2020. 244 p.

18. Ushakov I. V., Feodorov V. A., Permyakova I. J. Mechanical characteristics and crystallization of annealed metallic glass 82K3XCP. Proceedings of SPIE - The International Society for Optical Engineering. 2004. Vol. 5400. pp. 261-264.

19. Yu M.-H. Unified strength theory and its applications. Springer. 2018. 463 p.

20. Faraji G., Kim H. S., Kashi H. T. Severe plastic deformation: Methods, processing and properties. Elsevier. 2018. 324 p.

21. Silberschmidt V. V. Mechanics of materials in modern manufacturing methods and processing techniques. Elsevier. 2020. 465 p. 